\title{
Reproducibility of linear cardiac output measurement by Doppler ultrasound alone
}

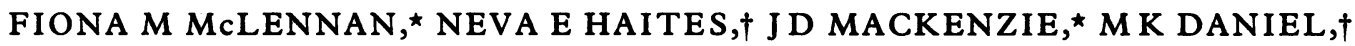 \\ JM RAWLES $\dagger$
}

From the $\star$ Department of Anaesthetics, Aberdeen Royal Infirmary and $\dagger$ Department of Medicine, University of Aberdeen, Foresterhill, Aberdeen

SUMMARY Inclusion of a pig aorta in an artificial circulation with pulsed blood flow allowed correlation of minute distance, measured in the aorta by Doppler ultrasound, and absolute blood flow, measured by timed blood-volume collection. The correlation coefficient was 0.99 with a standard error of prediction that was $5.4 \%$ of the minute distance predicted at a standard flow rate of 5 litres per minute. The horizontal distance between $95 \%$ confidence limits for a single prediction expressed as a percentage of 5 litres per minute was $33 \%$, and this corresponded to the range of flow rates of 1.65 litres per minute that could give rise to the same measurement.

In 142 patients duplicate measurements of minute distance were made with repositioning of the ultrasound transducer between recordings. The mean difference between paired readings, expressed as a percentage of the average (SD) of each pair was $5.4(4.7) \%$. Thus, the non-invasive measurement of linear cardiac output by Doppler ultrasound is similarly reproducible in vitro and in vivo and compares favourably with the measurement of volumetric cardiac output by thermodilution.

Non-invasive methods of measuring cardiac output using Doppler ultrasound have recently become available, ${ }^{12}$ leading to the development of the concept of cardiac output as a distance. ${ }^{3}$ Before measurement of linear cardiac output can be used with confidence, however, comparison with conventional volumetric cardiac output is needed. A newly introduced technique even if it is intrinsically highly reproducible may appear to perform badly if compared with a reference method that is unreliable. ${ }^{4}$ We have described a method of assessing the accuracy and reproducibility of blood flow measurement by thermodilution using an artificial circulation in which absolute flow rate can be measured. ${ }^{5}$ From the regression between the measurement of blood flow rate and its absolute value, the horizontal distance between the $95 \%$ confidence limits of a single prediction was calculated. This represented the range of flow rates that could give rise to an identical measurement; a change of flow rate has to exceed this figure to give rise to a change of measurement with $95 \%$ certainty. In this paper we describe how by incorporation of a

Requests for reprints to Dr John M Rawles, University Department of Medicine, Aberdeen Royal Infirmary, Foresterhill, Aberdeen AB9 2ZB.

Accepted for publication 17 September 1985 pig aorta into the artificial circulation we were able to correlate absolute and Doppler ultrasound measurement of blood flow to give an assessment of the latter method's reproducibility and ability to detect a change of flow rate in terms directly comparable with thermodilution.

The reproducibility of linear cardiac output measurement made by Doppler ultrasound alone was also assessed from paired recordings in patients.

\section{Methods}

DOPPLER MEASUREMENT IN VITRO

An aorta was dissected from a large pig and after its side vessels had been tied off it was incorporated at point " $C$ " into the artificial circulation previously described. ${ }^{5}$ The aorta was allowed to assume its natural curvature while suspended in a dish of liquid paraffin. The ultrasound transducer was aligned tangentially to the arch and in line with the direction of midstream blood flow and recordings made at seven different pulsatile flow rates.

DOPPLER MEASUREMENT IN VIVO

In 142 subjects aortic blood velocity was recorded over an average of 11.7 cardiac cycles using the Transcutaneous Aortic Velograph (TAV) Type 


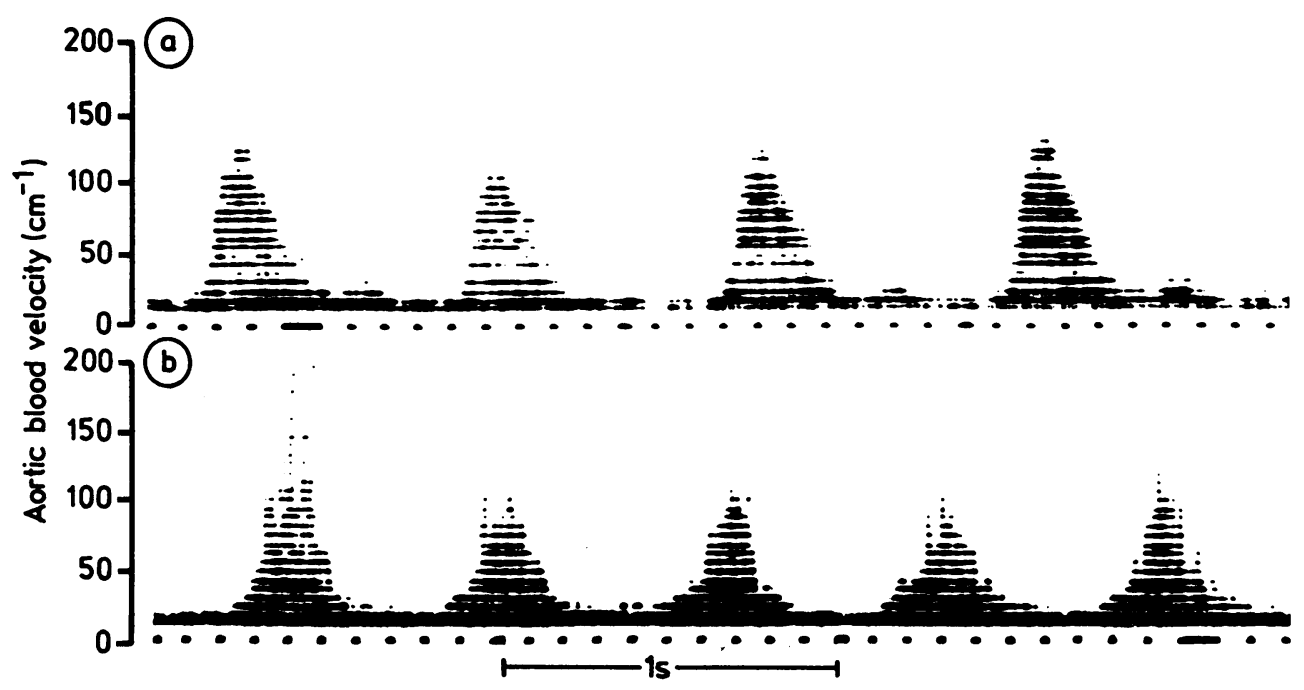

Fig. 1 Doppler ultrasound recordings from a normal subject ( $a$ ) and a pig aorta in an artificial circulation (b).

1006 (Muirhead Medical Ltd) as described previously. ${ }^{6}$ The recordings were made by one operator (FM McC) with the subject supine or in the left lateral position and they were then immediately repeated after removing and repositioning the transducer.

\section{INTERPRETATION OF DOP PLER RECORDINGS}

Fig. 1 shows a typical in vivo trace (a) and (b) shows how similar it is to an in vitro trace. The velocity-time complexes are approximately triangular, and their outline represents the highest velocity recorded in the ultrasound path at any instant. The area within the velocity-time complex is recorded in $\mathrm{cm}$ as stroke distance, with the product of stroke distance and heart rate giving minute distance. For each in vivo recording peak aortic blood velocity and stroke and minute distances were averaged from 10-12 consecutive beats using our own curve-following programme with a Summagraphics Bitpad and Sirius microcomputer. The coefficient of variation of each of these measurements was also computed to show the extent of beat-to-beat variation. A mean of 17 consecutive pulses from each in vitro recording was digitised at each flow rate.

\section{REPRODUCIBILITY OF DIGITISING DOPPLER RECORDINGS}

Sixty aortovelography recordings were digitised by the same person (MKD) on two occasions that were separated by an interval of several weeks and the results were compared. The electrocardiogram was not recorded and heart rate was estimated from the mean interval between the leading edges of consecutive velocity-time complexes. Another 50 aortovelography recordings were digitised twice by a second person $(\mathrm{NEH})$.

\section{STATISTICAL METHODS}

We used linear regression to relate actual blood flow and its measurement and to calculate the regression equation that was used to predict the measurement ( \pm the standard error of prediction) that would be shown at a standard flow rate of 5 litres per minute. ${ }^{7}$ The graphical results show the regression line together with the $95 \%$ confidence limits for a single prediction. The change of blood flow rate equivalent to the horizontal distance between the confidence limits was calculated and expressed as a percentage of 5 litres per minute.

The reproducibility of paired measurements was calculated as the difference between the greater and lesser measurements expressed as a percentage of the mean of the pair.

\section{Results}

COMPARISON OF IN VITRO DOPPLER AND

FLOW RATE

Figure 2 shows the correlation between absolute flow rate and minute distance determined ultrasonically in a pig aorta included in the artificial circulation. The minute distance at each point on the regression line was derived from an average of 17 systolic velocity-time complexes and the standard 


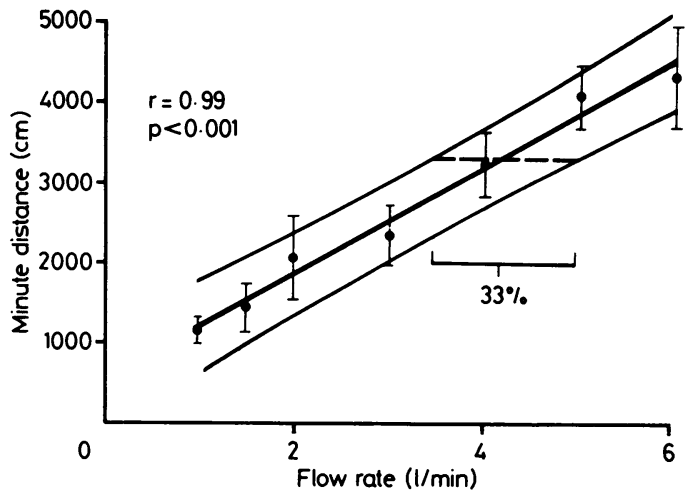

Fig. 2 Regression line and $95 \%$ confidence limits of a single prediction for the measurement of minute distance against flow rate in a pig aorta in an artificial circulation. At each flow rate the mean ( $S E$ ) of an average of $17 \cdot 7$ beats is indicated. Horizontal distance between confidence limits is expressed as a percentage of standard flow rate of 5 litres per minute.

error of each mean is indicated; the mean pulse rate was 98 beats per minute. The correlation coefficient is 0.99 and the regression equation is $\mathrm{y}=572+$ $2.36 \mathrm{x}$. The standard error of the prediction at a flow rate of 5 litres per minute is $209 \mathrm{~cm}$. This cannot be directly compared with flow rate but is $5.4 \%$ of the minute distance of $3850 \mathrm{~cm}$ predicted for a standard flow rate of 5 litres per minute. The $95 \%$ confidence limits at that flow rate are $\pm 14 \%$, and the horizontal distance between confidence limits expressed as a percentage of the standard flow rate is $33 \%$ of the standard flow rate or 1.65 litres per minute.

Table 1 Mean beat-to-beat coefficient of variation and reproducibility (SD) of aortic blood velocity measurements in 142 paired recordings in vivo. The average number of complexes in each recording was $11 \cdot 7(2 \cdot 1)$

\begin{tabular}{lll}
\hline & $\begin{array}{l}\text { Coefficient of variation } \\
(\%)\end{array}$ & $\begin{array}{l}\text { Reproducibility } \\
(\%)\end{array}$ \\
\hline Peak velocity & $4 \cdot 2(1 \cdot 4)$ & $3 \cdot 6(2 \cdot 8)$ \\
Heart rate & $5 \cdot 7(2 \cdot 3)$ & $4 \cdot 1(3 \cdot 7)$ \\
Stroke distance & $8 \cdot 7(2 \cdot 5)$ & $5 \cdot 2(4 \cdot 6)$ \\
Minute distance & $8 \cdot 1(2 \cdot 8)$ & $5 \cdot 4(4 \cdot 7)$ \\
\hline
\end{tabular}

Table 2 Mean reproducibility (SD) of digitising aortic blood velocity recordings on two occasions by two observers

\begin{tabular}{lll}
\hline & Observer 1 & Observer 2 \\
\hline Peak velocity & $5 \cdot 0(7 \cdot 1)$ & $4.6(5 \cdot 3)$ \\
Heart rate & $0.9(0 \cdot 7)$ & $0.9(2 \cdot 3)$ \\
Stroke distance & $5 \cdot 5(7 \cdot 5)$ & $5 \cdot 3(4 \cdot 8)$ \\
Minute distance & $5 \cdot 7(7 \cdot 1)$ & $4.8(4.5)$ \\
\hline
\end{tabular}

\section{REPRODUCIBILITY OF IN VIVO DOPPLER} MEASUREMENTS

Table 1 summarises the reproducibility of peak aortic blood velocity, heart rate, and stroke and minute distances in 142 paired measurements. The mean reproducibility of minute distance was $5.4 \%$, but some of this variability arises from spontaneous fluctuation of cardiac output rather than its measurement. The mean reproducibility of heart rate (which is measured with an accuracy of $>99 \%$ ) was $4.1 \%$, and the correlation between differences in minute distance and differences in heart rate had a coefficient of $0.4(\mathrm{p}<0.001)$; the proportion of variance in paired minute distance measurements that may be attributed to changes in heart rate is therefore at least $16 \%$.

There is no consistent trend between the first and second measurements, except for peak aortic blood velocity which fell by $<1 \%(p<0.05)$. Heart rate increased by $<1$ beat per minute $(p>0.05)$. The mean beat-to-beat coefficient of variation is least for peak aortic blood velocity and greatest for stroke distance and is less than $10 \%$ in all cases.

\section{REPRODUCIBILITY OF DIGITISING DOPPLER RECORDINGS}

Some of the differences between paired measurements could arise when the recordings are digitised and variability from this source is indicated in Table 2. Reproducibility is summarised for peak aortic blood velocity, heart rate, and stroke and minute distances for two observers who respectively digitised 50 and 60 recordings on two occasions. Reproducibility of heart rate measurements was < $1 \%$, and that of the stroke and minute distances was about $5 \%$.

\section{Discussion}

MEASUREMENT OF CARDIAC OUTPUT BY DOPPLER ULTRASOUND AND ECHOCARDIOGRAPHY

Several groups have shown that it is possible to measure left ventricular stroke volume, and hence cardiac output, non-invasively using the combination of Doppler ultrasound and echocardiography. ${ }^{18-10}$ Stroke volume is calculated as the product of stroke distance and aortic cross sectional area. Stroke distance is not measured as such but can be calculated as the systolic-velocity integral at a point where the ultrasound beam is in line with the direction of flow. Ultrasound access from the suprasternal notch permits the measurement of stroke distance in the aortic root or in the aortic arch, which is the site we use, similar values being obtained at both sites. ${ }^{1112}$ The product 
of stroke distance and heart rate gives minute distance; stroke and minute distance are linear equivalents of stroke volume and cardiac output respectively.

There are three assumptions implicit in the method. Firstly, that stroke distance used in the calculation is representative of blood flow as a whole, as it would be only if the flow profile were flat. Since the flow profile in the aorta is skewed this assumption is only approximate. The second assumption is that total stroke volume passes the point at which stroke distance is measured. This too is not the case since a proportion of stroke volume enters the coronary circulation from the aortic root and a larger proportion is lost to the head and neck before velocity is measured in the aortic arch. The third assumption is also unwarranted; this is that the aortic cross section has a regular geometrical shape whose area can be readily calculated and that this remains constant throughout the cardiac cycle. Nevertheless, despite these three approximations it has been shown that there is close correspondence between stroke volumes determined in this way and by conventional invasive methods.

MEASUREMENT OF CARDIAC OUTPUT BY DOPPLER ULTRASOUND ALONE

Thus, stroke volume approximates to the product of stroke distance and aortic cross sectional area or stroke distance approximates to stroke volume divided by aortic cross sectional area. Stroke distance is therefore related to stroke index; both are unidimensional, and both are corrected for variation in body size; the former in effect by division of stroke volume by aortic cross sectional area, the latter by division by body surface area. Stroke distance would therefore be expected to be proportional to stroke volume within subjects, ${ }^{13-16}$ to be independent of body size, ${ }^{2}$ and its product with heart rate (that is minute distance) should provide an absolute measure of cardiac output. ${ }^{6}$ All three expectations of the measurement have been fulfilled. Use of this linear measure to assess cardiac output obviates the need to measure aortic cross sectional area which is technically the most demanding part of the procedure and the part which is associated with the greatest error. ${ }^{12}$ When linear cardiac output alone is calculated and compared with a normal range of values none of the three assumptions involved in conversion of velocity measurements to volume is made. ${ }^{3}$

Whereas the weakness of thermodilution is its performance during pulsatile flow, ${ }^{5}$ the strength of the Doppler ultrasound method of measuring cardiac output that we describe is its ability to measure beat-to-beat changes of velocity. The beat-to-beat coefficient of variation ranges from $4.2 \%$ for peak aortic blood velocity to $8.7 \%$ for stroke distance. Thus for a recording of 10 beats the standard error of the mean for stroke distance is $2.8 \%$ falling to $1.9 \%$ for a recording of 20 beats. For most purposes a 10 beat recording provides a sufficiently accurate estimate of minute distance. Distante et al using aortovelography found a beat-to-beat coefficient of variation for time averaged velocity (corresponding to minute distance $/ 60$ ) of $10 \%{ }^{16}$

\section{REPRODUCIBILITY OF DOP PLER ULTRASOUND MEASUREMENTS}

The main sources of imprecision in the measurement of linear cardiac output are alignment of the ultrasound beam and the signal to noise ratio. The measured velocity of blood flow is proportional to the cosine of the angle between the direction of the ultrasound beam and the direction of blood flow. Since the cosine of an angle $<18^{\circ}$ is $>0.95$ there is an angular latitude of $36^{\circ}$ for $<5 \%$ underestimation of velocity. The transcutaneous aortovelograph used by us generates a broad beam of continuous ultrasound which detects movement at any distance from the transducer, subject only to the attenuation of ultrasound by transmission through tissue. By recording velocities in the aortic arch correct positioning of the transducer is assured since axial flow is almost certain to be aligned with the ultrasound beam at some point around the arch. Correct alignment is aided by selecting the position which gives the highest pitch of audible Doppler shift frequencies and the greatest velocity on the on-line paper record.

Recognition of the maximum velocity of movement within the ultrasound beam may be achieved by electronic signal processing that provides an analogue signal proportional to the greatest frequency shift detected at any moment. ${ }^{17}$ This method, however, does not differentiate between forward or reverse flow and is prone to artefactual error. Spectral analysis of the signal is more satisfactory since this gives the velocity spectrum and allows recognition of turbulence and separation of forward and reverse flow. Our equipment uses spectral analysis, the amplitude of each frequency band (and hence velocity) being recorded on a grey scale by a separate pen. Reproducible recognition and integration of maximal velocity requires accurate edge detection of the velocity-time complex which depends on a satisfactory signal to noise ratio.

The leading edges of the velocity-time complexes recorded by aortovelography are easily recognised and the distance from one leading edge to the next is used to calculate mean heart rate. Reproducibility of 
mean heart rate in paired digitised measurements of the same records was $<1 \%$. Peak aortic blood velocity, marked by the apex of the triangular shaped velocity-time complex, had a digitising reproducibility of about $5 \%$, and that of stroke distance, measured as the area of the triangle, was $<6 \%$. In paired recordings the worst reproducibility of any measurement was $5.4 \%$ for minute distance in which imprecision in the measurements of flow time, peak aortic blood velocity, heart rate, and the process of digitising are all compounded. Peak aortic blood velocity was the most reproducible measurement, with an average difference of $4 \%$ between paired readings (Table 2 ).

This degree of reproducibility of aortovelography in vivo is consistent with that found by us in vitro. The $95 \%$ confidence limits for minute distance at a flow rate of 5 litres per minute in vitro were $\pm 14 \%$. The $95 \%$ confidence limits for reproducibility in vivo are about $15 \%$, there being little difference between the two figures despite short term changes in flow rate and repositioning the transducer between measurements in vivo. Fraser et al measured mean blood velocity in adults by aortovelography with reproducibility of $6.4 \%$ with the same observer and $7 \cdot 4 \%$ with different observers. ${ }^{18}$ Using a pulsed Doppler system Gisvold and Brubakk obtained a coefficient of variation within subjects of $6-11 \%$ for all velocity measurements in the aortic arch. ${ }^{11}$

Gardin et al used a -range-gated Doppler ultrasound technique to measure blood flow in the ascending aorta. ${ }^{19}$ Reproducibility of measurement of the Doppler records was $2.5 \%$ for peak aortic blood velocity, $1.9 \%$ for ejection time, and $3.2 \%$ for the flow velocity integral or stroke distance, the latter being calculated from electronic measurement of the former without planimetry. Day to day reproducibility was $3.8(3.1) \%$ for stroke distance. Hatle and Angelsen reported that the reproducibility of the integral under the maximal velocity curve was $9.1 \%$ in paired measurements separated by days or months. ${ }^{20}$

\section{MEASUREMENT OF A CHANGE OF CARDIAC \\ OUTPUT}

The most important clinical application of cardiac output measurement is the recognition of a change, particularly a fall, of cardiac output. A change of cardiac output may be measured under three circumstances. Firstly, when any number of measurements may be made before and after an intervention. The ability to detect a change is then only limited by the number of measurements made before and after its occurrence. Secondly, a sufficient number of measurements may have been made before the change to establish a mean cardiac output and its standard deviation. A single measurement taken after an intervention that is more than two standard deviations from the previously established mean may be taken to have resulted from a true haemodynamic change. The third situation is when the standard deviation of the current series of measurements is not known and has to be assumed to be similar to previous experience. Two single measurements can only be assumed to have resulted from a changed cardiac output if they differ by more than four standard deviations. This is the situation described in this and the preceding paper. $^{5}$

In comparing the performance of different methods of measuring cardiac output the standard deviation of the measurement is inadequate by itself-what is important is the variation of flow rate which gives rise to a change of measurement. This is why we have suggested the horizontal distance between confidence limits since this combines a measure of variability of the measurement, that is the confidence limits of a single prediction, with the slope of the regression line which relates flow rate and its measurement. From a reproducibility study alone the extent of the haemodynamic change that has given rise to a change in measurement of flow rate cannot be known without an independent measure of blood flow.

In vitro the range of blood flow rate that could give rise to an identical measurement by Doppler ultrasound was 1.65 litres per minute, that is $33 \%$ of the standard flow rate. A greater change of flow rate than this would be necessary for $95 \%$ certainty of a difference between two single measurements. This compares with $21-33 \%$ for three different makes of thermodilution cardiac output computer under optimal conditions in vitro, and $30-62 \%$ overall. The percentage vertical spread of the confidence limits for aortovelography is similar to that of the cardiac output computer with the least variability but the horizontal distance between confidence limits depends also on the slope of the regression line. All of the regression lines for cardiac output computers had slopes $>1$ and passed below the origin whereas for aortovelography the slope is $<1$ and passes above the origin. This has the effect of reducing the sensitivity of aortovelography to measure a change of flow rate compared with thermodilution where overestimation of absolute flow rate increases the ability to detect change.

\section{EFFECT OF BLOOD PRESSURE ON AORTIC}

BLOOD VELOCITY

In our circulation model blood pressure was not controlled, so that as flow rate was reduced blood 
pressure fell resulting in reduction of the cross sectional area of the pig aorta and a less than proportionate fall of velocity and the velocity-integral. Thus, when a flow rate of 5 litres per minute was halved, minute distance was reduced to $57 \%$ of the initial value; the predicted value of minute distance for zero flow was $572 \mathrm{~cm}$. Light has calculated that if in man the same reduction of cardiac output from 5 to 2.5 litres per minute is accompanied by a fall of systolic blood pressure of $45 \mathrm{~mm} \mathrm{Hg}$, aortic blood velocity would fall to $58 \%$ of its previous value. ${ }^{21}$ This suggests that the compliance of the pig aorta used in vitro is similar to that of the aorta in intact man.

The extent of the influence of blood pressure on aortic blood velocity needs clarification. In hypertensive patients aortic blood velocity is reduced compared with normal controls, ${ }^{6}$ but this could be due either to an increased aortic arch diameter ${ }^{22}$ or to reduced cardiac output, ${ }^{23}$ both of which have been demonstrated in hypertension. In non-hypertensive normal subjects aortic blood velocity was independent of blood pressure. ${ }^{2}$ In dogs aortic diameter correlated with cardiac output, but the graph relating cardiac output (measured as the product of aortic cross sectional area and systolic velocity integral) and absolute flow rate went virtually through the origin. ${ }^{12}$ It seems likely then that fluctuation of blood pressure within the normal range has little effect on aortic blood velocity, but the ability of aortovelography to detect a fall of cardiac output is slightly reduced by any large accompanying fall of blood pressure.

\section{CONCLUSIONS}

Cardiac output is the ultimate expression of cardiovascular function and there are many clinical circumstances where its measurement is desirable. The standard technique for measuring cardiac output is thermodilution which is technically difficult, costs about $£ 100$ per patient in disposable items ${ }^{24}$ and carries a risk of serious complications. ${ }^{25}$ Our experiments have shown that under optimum conditions of pulsatile flow in vitro thermodilution provides an unreliable estimate of absolute flow rate and detects $21-33 \%$ changes of flow rate with $95 \%$ certainty and with a reproducibility that is seldom better than $10 \%$. The method is unlikely to perform as well as this under clinical conditions.

The non-invasive measurement of linear cardiac output by Doppler ultrasound is simple, without risk or discomfort, and has negligible running costs. In vitro the method detects a 33\% change of flow rate with $95 \%$ certainty. In the absence of aortic disease the absolute value of linear cardiac output is meaningful, ${ }^{6}$ and under clinical conditions its measurement has a reproducibility of about $5 \%$.

F M McL is research fellow of the Association of Anaesthetists of Great Britain and Ireland. M KD was supported by the Scottish Home and Health Department with a Medical Student Vacation Research Grant. We are also grateful to Grampian Health Board for a research grant.

\section{References}

1 Huntsman LL, Stewart DK, Barnes SR, Franklin SB, Colocousis JS, Hessel EA. Noninvasive Doppler determination of cardiac output in man. Clinical validation. Circulation 1983; 67: 593-602.

2 Mowat DHR, Haites NE, Rawles JM. Aortic blood velocity measurement in adults using a simple ultrasound technique. Cardiovasc Res 1983; 17: 75-80.

3 Haites NE, McLennan FM, Mowat DHR, Rawles JM. How far is the cardiac output? Lancet 1984; ii: 1025-7.

4 Schuster AH, Nanda NC. Doppler echocardiographic measurement of cardiac output: comparison with a nongolden standard. Am $\mathcal{f}$ Cardiol 1984; 53: 257-9.

5 Mackenzie JD, Haites NE, Rawles JM. Method of assessing the reproducibility of blood flow measurement: factors influencing the performance of thermodilution cardiac output computers. Br Heart $\mathcal{F} 1986$; 55: 14-24.

6 Haites NE, McLennan FM, Mowat DHR, Rawles JM. Assessment of cardiac output by the Doppler ultrasound technique alone. Br Heart $\mathcal{f}$ 1985; 53: 123-9.

7 Snedecor GW, Cochran WG. Statistical methods, 7th ed. Iowa: The Iowa State University Press, 1980: 166.

8 Magnin PA, Stewart JA, Myers S, et al. Combined Doppler and phased array echocardiographic estimation of cardiac output. Circulation 1981; 63: 388-92.

9 Goldberg SJ, Sahn DJ, Allen HD, Valdes-Cruz LM, Hoenecke H, Carnahan Y. Evaluation of pulmonary and systemic blood flow by 2-dimensional Doppler echocardiography using fast Fourier transform spectral analysis. Am $\mathcal{F}$ Cardiol 1982; 50: 1394-400.

10 Chandraratna PA, Nanna M, McKay C, et al. Determination of cardiac output by transcutaneous continuous-wave ultrasonic Doppler computer. $A m$ Heart $\mathcal{F}$ 1984; 53: 234-7.

11 Gisvold SE, Brubakk AO. Measurement of instantaneous blood-flow velocity in the human aorta using pulsed Doppler ultrasound. Cardiovasc Res 1982; 16: 26-33.

12 Fisher DC, Sahn DJ, Friedman MJ, et al. The effect of variations on pulsed Doppler sampling site on calculation of cardiac output: an experimental study in open chest dogs. Circulation 1983; 67: 370-6.

13 Light LH, Cross G. Convenient monitoring of cardiac output and global left ventricular function by transcutaneous aortovelography -an effective alternative to cardiac output measurements. In: Spencer M, ed. Cardiac Doppler diagnosis. The Hague: Martinus Nyhoff, 1983: 69.

14 Sequeira RF, Light LH, Cross G, Raftery EB. Trans- 
cutaneous aortovelography. A quantitative evaluation. Br Heart ₹ 1976; 38: 443-50.

15 Bilton AH, Brotherhood J, Cross B, Hanson GC, Light LH, Sequeira RF. Transcutaneous aortovelography as a measure of central blood flow. F Physiol (Lond) 1978; 281: 4-5P.

16 Distante A, Moscarelli E, Rovai D, L'Abbate A. Monitoring changes in cardiac output by transcutaneous aortovelography a non-invasive Doppler technique: comparison with thermodilution. $\mathcal{F} \mathrm{Nucl}$ Med Allied Sci 1980; 24: 171-5.

17 Ihlen H, Amlie JP, Dale J, et al. Determination of cardiac output by Doppler echocardiography. Br Heart f 1984; 51: 54-60.

18 Fraser CB, Light LH, Shinebourne EA, Buchtal A, Healy MJR, Beardshaw JA. Transcutaneous aortovelography: reproducibility in adults and children. Eur f Cardiol 1976; 4/2: 181-9.

19 Gardin JM, Dabestani A, Matin K, Allfie A, Russell.D,
Henry WL. Reproducibility of Doppler aortic blood flow measurements: studies on intraobserver, interobserver and day-to-day variability in normal subjects. Am $\mathcal{F}$ Cardiol 1984; 54: 1092-8.

20 Hatle L, Angelsen B. Doppler ultrasound in cardiology. Physical principles and applications. Philadelphia: Lea and Febiger, 1982: 192.

21 Light $H$. Transcutaneous aortovelography. A new window on the circulation? Br Heart $\mathcal{f}$ 1976; 38: 433-42.

22 Goldberg BB. Suprasternal ultrasonography. $\mathcal{F} A M A$ 1971; 215: 245-50.

23 Lund-Johansen P. Central haemodynamics in essential hypertension. Acta Med Scand [Suppl] 1977; 606: 35-42.

24 Bayliss J, Norell M, Ryan A, Thurston M, Sutton GC. Bedside haemodynamic monitoring: experience in a general ward. $\mathrm{Br} \mathrm{Med}$ f 1983; 287: 187-90.

25 Anonymous. Complications of pulmonary artery balloon flotation catheters. Lancet 1983; i: 37. 